\title{
Prognostic Stage IIIA Breast Cancer AJCC v8
}

National Cancer Institute

\section{Source}

National Cancer Institute. Prognostic Stage IIIA Breast Cancer A/CC v8. NCI Thesaurus. Code C139583.

Stage IIIA includes: (T 0-1, N1, M0, G2, HER2 Status: Neg ative, ER Status: Negative, PR Status: Negative); (T0-1, N1, M0, G3, HER2 Status: Negative, ER Status: Positive, PR Status: Negative); (T0-1, N1, M0, G3, HER2 Status: Negative, ER Status: Negative, PR Status: Any); (T2, NO, M0, G2, HER2 Status: Negative, ER Status: Negative, PR Status: Negative); (T2, N0, M0, G3, HER2 Status: Negative, ER Status: Positive, PR Status: Negative); (T2, N0, M0, G3, HER2 Status: Negative, ER Status: Negative, PR Status: Any); (T2, N1, M0, G1, HER2 Status: Negative, ER Status: Positive, PR Status: Negative); (T2, N1, M0, G2, HER2 Status: Positive, ER Status: Negative, PR Status: Negative); (T2, N1, M0, G2, HER2 Status: Negative, ER Status: Positive, PR Status: Negative); (T2, N1, M0, G3, HER2 Status: Positive, ER Status: Positive, PR Status: Negative); (T2, N1, M0, G3, HER2 Status: Positive, ER Status: Negative, PR Status: Negative); (T3, N0, M0, G1, HER2 Status: Neg ative, ER Status: Positive, PR Status: Negative); (T3, NO, M0, G2, HER2 Status: Positive, ER Status: Negative, PR Status: Negative); (T3, N0, M0, G2, HER2 Status: Negative, ER Status: Positive, PR Status: Negative); (T3, N0, M0, G3, HER2 Status: Positive, ER Status: Positive, PR Status: Negative); (T3, N0, M0, G3, HER2 Status: Positive, ER Status: Negative, PR Status: Negative); (T0-2, N2, M0, G1, HER2 Status: Positive, ER Status: Positive, PR Status: Neg ative); (T 0-2, N2, M0, G1, HER2 Status: Positive, ER Status: Negative, PR Status: Any); (T0-2, N2, M0, G1, HER2 Status: Negative, ER Status: Positive, PR Status: Negative); (T0-2, N2, M0, G1, HER2 Status: Negative, ER Status: Negative, PR Status: Positive); (T0-2, N2, M0, G2, HER2 Status: Positive, ER Status: Positive, PR Status: Negative); ( T0-2, N2, M0, G2, HER2 Status: Positive, ER Status: Negative, PR Status: Any); (T3, N1-2, M0, G1, HER2 Status: Positive, ER Status: Positive, PR Status: Negative); (T3, N1-2, M0, G1, HER2 Status: Positive, ER Status: Negative, PR Status: Any); (T3, N1-2, M0, G1, HER2 Status: Negative, ER Status: Positive, PR Status: Negative); (T3, N1-2, M0, G1, HER2 Status: Negative, ER Status: Neg ative, PR 
Status: Positive); (T3, N1-2, M0, G2, HER2 Status: Positive, ER Status: Positive, PR Status: Negative); (T3, N1-2, M0, G2, HER2 Status: Positive, ER Status: Neg ative, PR Status: Any); (T4, N0-2, M0, G1, HER2 Status: Negative, ER Status: Positive, PR Status: Positive); (Any T, N3, M0, G1, HER2 Status: Negative, ER Status: Positive, PR Status: Positive). T0: No evidence of primary tumor. T1: Tumor measuring $20 \mathrm{~mm}$ or less in greatest dimension. T2: Tumor measuring more than $20 \mathrm{~mm}$, but not more than $50 \mathrm{~mm}$ in greatest dimension. T3: T umor measuring more than $50 \mathrm{~mm}$ in greatest dimension. T4: Tumor of any size with direct extension to the chest wall and/or to the skin (ulceration or macroscopic nodules); invasion of the dermis alone does not qualify as T4. N0: No regional lymph node metastasis is identified or isolated tumor cell clusters (ITCS) are identified only. N1: T umor with micrometastases; or metastases in 1-3 axillary lymph nodes; and/or clinically negative internal mammary nodes with micrometastases or macrometastases by sentinel lymph node biopsy. N2: Tumor with metastases in 4-9 axillary lymph nodes; or positive ipsilateral internal mammary lymph nodes by imag ing in the absence of axillary lymph node metastases. N3: Tumor with metastases in 10 or more axillary lymph nodes; or in infraclavicular (level III axillary) lymph nodes; or positive ipsilateral internal mammary lymph nodes by imaging in the presence of one or more positive level I, II axillary lymph nodes; or in more than three axillary lymph nodes and micrometastases or macrometastases by sentinel lymph node biopsy in clinically negative ipsilateral internal mammary lymph nodes; or in ipsilateral supraclavicular lymph nodes. G1: Low combined histologic grade (favorable); SBR score of 3-5 points. G2: Intermediate combined histologic grade (moderately favorable); SBR score: 6-7 points. G3: High combined histologic grade (unfavorable); SBR score of 8-9 points. M0: No clinical or radiographic evidence of distant metastases. Imaging studies are not required to assign the MO category. (AJCC 8th ed.) 